\title{
ORIGINAL ARTICLE THE PREDICTIVE UTILITY OF GRACE SCORE FOR OBSTRUCTIVE CORONARY ARTERY DISEASE IN PATIENTS WITH NON ST ELEVATION MYOCARDIAL INFARCTION
}

\author{
Haroon Ishaq ${ }^{1}$, Bilal Akhtar1, Mukesh Kumar ${ }^{1}$, Ghulam Shabbir Shar', Abdul Hakeem', \\ Sobia Masood ${ }^{1}$, Tahir Saghir ${ }^{1}$, Musa Karim ${ }^{1}$ \\ ${ }^{1}$ National Institute of Cardiovascular Diseases, Karachi, Pakistan
}

\begin{abstract}
Objectives: The objective of this study was to determine the predictive value of GRACE score for predicting obstructive coronary artery disease in patients with non ST-segment elevation myocardial infarction (NSTEMI).

Methodology: This cross-sectional study was conducted at the largest public sector cardiac care center of the Pakistan between January 2020 and June 2020. In this study, we included adult patients diagnosed with NSTEMI and correlation of GRACE score was assessed with angiographic finding of obstructive CAD defined as $\geq 50 \%$ stenosis in the left main or $\geq 70 \%$ stenosis in other coronary arteries.

Results: A total of 227 patients were included in this study, out of whom $72.2 \%$ (164) were male patients and mean age was $55.77 \pm 9.15$ years. Mean GRACE score was found to be 95.89 \pm 21.15 . On coronary angiography obstructive CAD was present in $84.6 \%$ (192) of the patients. Area under the cure for predicting obstructive CAD was 0.669 [0.552 to 0.785]. The optimal cutoff value of GRACE score was $\geq 84$ with sensitivity of $79.7 \%$ [73.3\% to $85.1 \%$ ] and specificity of $57.1 \%$ [39.3\% to $73.7 \%$ ]. GRACE score of $\geq 84$ was found to be an independent predictor of obstructive CAD with odds ratio of 4.33 [1.61 - 11.64; $\mathrm{p}=0.004]$ adjusted for gender, age, hypertension, diabetes, family history of CAD, and smoking.

Conclusion: GRACE score has a moderate predictive value in predicting obstructive CAD in patients with NSTEMI. The optimal cutoff value of 84 is an independent predictor with good sensitivity but moderate specificity in predicting obstructive CAD.

Keywords: coronary artery disease, acute myocardial infarction, non ST-segment elevation myocardial infarction, GRACE score, risk stratification, obstructive coronary artery disease
\end{abstract}

Citation: Ishaq H, Akhtar B, Kumar M, Shar GS, Hakeem A, Masood S, Saghir T, Karim M. The Predictive Utility of GRACE Score for Obstructive Coronary Artery Disease in Patients with Non ST Elevation Myocardial Infarction. Pak Heart J. 2021;54(03):239-243. DOI: https://doi.org/10.47144/phj.v54i3.2162

\section{INTRODUCTION}

Coronary artery disease (CAD) is among leading causes of premature life losses around the world. Acute coronary syndrome (ACS) is the most common clinical manifestation of CAD comprises of acute myocardial infarction (AMI), with and without ST segment elevation, and unstable angina pectoris. ACS consisted of patients with heterogeneous clinical spectrum with substantially varying outcomes and hospital course. Hence, management guidelines recommend risk stratification at individualized level to determine the prognosis and to optimize the planning and management of the patients. ${ }^{1}$

Effective risk stratification of ACS patients, especially non ST-segment elevation myocardial infarction (NSTEMI), has become the cornerstone of management of the patients considering the varying prognosis and increased risk of re-hospitalization due to recurrence of myocardial infarction (MI). Various risk stratification modalities have been proposed notably Global Registry of Acute Coronary Events (GRACE) score, Framingham Risk score, and Thrombolysis in Myocardial Infarction (TIMI) score. ${ }^{1}$ Both European and American clinical practice gaudiness endorses use of either TIMI or GRACE score for risk stratification of short- and mid-term outcome of these patients..$^{2-4}$ However, clinical evidence and literature is very limited regarding use of these scores for the prediction of the extent and severity of CAD. A reliable prediction of coronary anatomy before any invasive assessment, such as coronary angiography, can have great implications in clinical decision making regarding timing and intensity of therapeutic management as well as interventions. Such strategy can help us in identifying patients requiring more aggressive management to improve outcomes or, conversely, to avoid unnecessary invasive procedures in patients with nonobstructive CAD. 
Although GRACE risk score is an important and validated modality for assessing prognosis but clinical evidence regarding its predictive utility for discriminating anatomical extent of CAD is scarce. Therefore, aim of this study was to determine the predictive value of GRACE score for predicting obstructive coronary artery disease in patients with non ST-segment elevation myocardial infarction (NSTEMI).

\section{METHODOLOGY}

This cross-sectional study was conducted at the largest public sector cardiac care center of the Pakistan between January 2020 and June 2020. Required number of consecutive patients fulfilling the inclusion criteria were included in this study. Consent for participation was obtained from all the patients and approval for the study was obtained from the ethical review committee of the institution. Inclusion criteria for the study were adult patients of either gender, between 18 to 80 years of age, diagnosed with non-ST segment elevation myocardial infarction (NSTEMI), and undergone coronary angiography. Patients who refused to participate in the study, patients with prior history of cardiac related surgery or intervention, previously diagnosed with obstructive coronary artery disease (CAD), current diagnosis of ST-elevation myocardial infarction (STEMI) or new left bundle branch block, and patients with chest pain of proven non-ischemic etiology (such as takotsubo syndrome or myocarditis) were excluded from the study.

Diagnosis of NSTEMI was made based on history, presentation, ECG findings, and cardiac enzymes. Demographic details and information regarding preexisting co-morbid conditions were obtained for all the patients. Global Registry for Acute Coronary Events (GRACE) score (2.0) was calculated based on age, heart rate, systolic blood pressure, Killip class, cardiac arrest, ST-segment deviation, serum creatinine, and initial cardiac biomarker status available at https://www.mdcalc.com/grace-acs-risk-mortalitycalculator. All the patients underwent routine coronary angiography and all the procedures were performed and interpreted by the consultant cardiologist. Obstructive CAD was defined as $\geq 50 \%$ stenosis in the left main or $\geq 70 \%$ stenosis in other coronary arteries. Routine management was unaltered and all the patients received management as per the guidelines and institutional protocol. Data were collected on a pre-defined structured proforma.

Sample size for the study was calculated considering the expected AUC of $0.59^{5}$ for GRACE score to predict obstructive coronary artery disease at $95 \%$ confidence level and 6\% margin of error. The minimum required sample size was calculated to be $n=167$. Data analysis were performed with the help of IBM SPSS version 21. Data points were summarized with appropriate summary statistics such as mean \pm standard deviation (SD) or frequency (\%). The predictive utility of GRACE score was assessed by performing the receiver operating characteristic (ROC) curves analysis taken GRACE score as test variable and dichotomous variable of presence or absence of obstructive CAD as state variable. Area under the curve (AUC) along with $95 \%$ confidence interval (CI) were computed and prediction value of GRACE score was deemed to be significant when the AUC was statistically different from 0.5 . The optimal cutoff value of GRACE score for the prediction of obstructive CAD was obtained using Youden's index and accuracy, sensitivity, specificity, positive predictive value, and negative predictive value at the computed cutoff value were calculated. Multivariate binary logistic regression analysis was performed taken dichotomous variable of presence or absence of obstructive CAD as dependent variable and demographic characteristics, co-morbid conditions and GRACE score as independent variables. Odds ratio (OR) along with 95\% CI was reported and pvalue $\leq 0.05$ was taken as significance criteria throughout the analysis.

\section{RESULTS}

A total of 227 patients were included in this study, out of whom $72.2 \%$ (164) were male patients and mean age was $55.77 \pm 9.15$ years. Most of the patients $(90.7 \%)$ where in Killip class I at presentation, there were only four patients (1.8\%) were in Killip class III and no patient was in Killip class IV. Most common pre-existing co-morbid condition was hypertension (84.6\%) followed by diabetes mellitus (39.6\%). Mean GRACE score was found to be $95.89 \pm 21.15$. Demographic and clinical characteristics are presented in Table 1.

Table 1: Demographic and clinical characteristics

\begin{tabular}{|l|c|}
\hline Characteristics & Total \\
\hline Total (N) & $\mathbf{2 2 7}$ \\
\hline Gender & $72.2 \%(164)$ \\
\hline Male & $27.8 \%(63)$ \\
\hline Female & $55.77 \pm 9.15$ \\
\hline Age (years) & $34.4 \%(78)$ \\
\hline$\leq 50$ years & $59.9 \%(136)$ \\
\hline 51 to 70 years & $5.7 \%(13)$ \\
\hline > 70 years & $153.03 \pm 45.09$ \\
\hline Height $(\mathbf{c m})$ & $71.95 \pm 11.21$ \\
\hline Weight $(\mathbf{k g})$ & \\
\hline KILLIP Class & \\
\hline
\end{tabular}




\begin{tabular}{|l|c|}
\hline I & $90.7 \%(206)$ \\
\hline II & $7.5 \%(17)$ \\
\hline III & $1.8 \%(4)$ \\
\hline IV & $0 \%(0)$ \\
\hline Heart rate (bpm) & $82.06 \pm 13.08$ \\
\hline Systolic blood pressure (mmHg) & $134.42 \pm 16.62$ \\
\hline Serum creatinine (ng/dL) & $1.04 \pm 0.31$ \\
\hline Risk factors \\
\hline Hypertension & $84.6 \%(192)$ \\
\hline Diabetes & $39.6 \%(90)$ \\
\hline Family history of CAD & $10.1 \%(23)$ \\
\hline Smoking & $18.5 \%(42)$ \\
\hline Obesity & $7.9 \%(18)$ \\
\hline GRACE Score & $95.89 \pm 21.15$ \\
\hline
\end{tabular}

$C A D=$ coronary artery diseases

On coronary angiography obstructive CAD was present in $84.6 \%$ (192) of the patients out of which $25.5 \%$ (49) had single vessel disease, $28.1 \%$ (54) had two vessel disease, and $46.4 \%$ (89) had three vessel disease. The ROC analysis (Figure 1) revealed significant predictive value of the GRACE score for predicting obstructive CAD with AUC of 0.669 [95\% CI; 0.552 to 0.785$]$. The optimal cutoff value of GRACE score was computed to be $\geq 84$ with overall all accuracy of $76.2 \%$ [95\% CI; $70.1 \%$ to $81.6 \%$ ] in discriminating obstructive CAD with sensitivity of $79.7 \%$ [95\% CI; $73.3 \%$ to $85.1 \%$ ] and specificity of $57.1 \%$ [95\% CI; $39.3 \%$ to $73.7 \%$ ]. Angiographic findings and diagnostic accuracy of GRACE score at the calculated optimal cutoff value are presented in Table 2.

Table 2: Angiographic findings and diagnostic accuracy of GRACE score

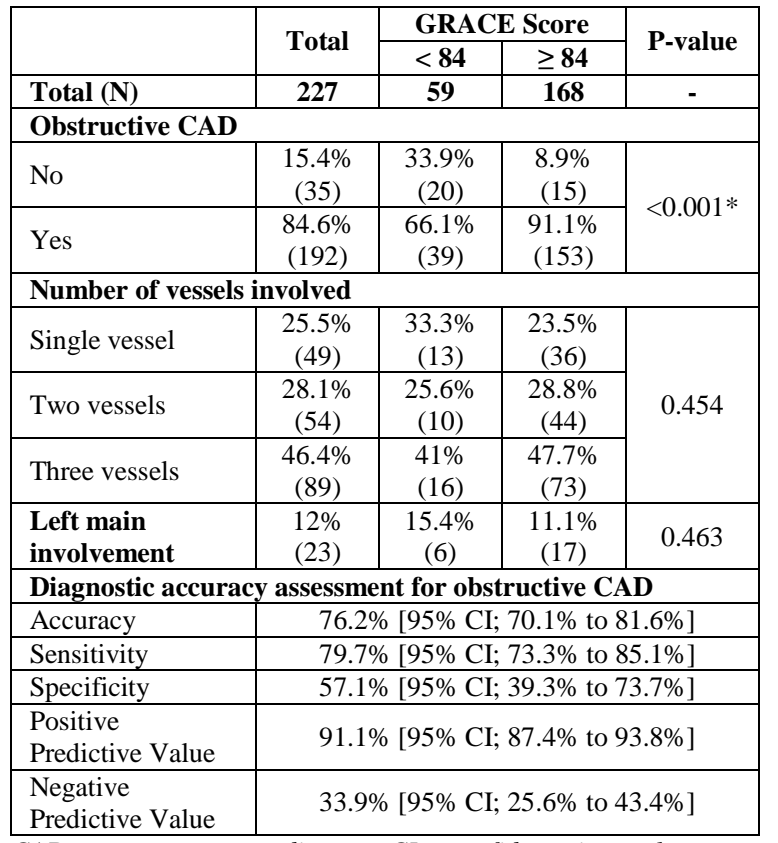

$C A D=$ coronary artery diseases, $C I=$ confidence interval

*significant at $5 \%$

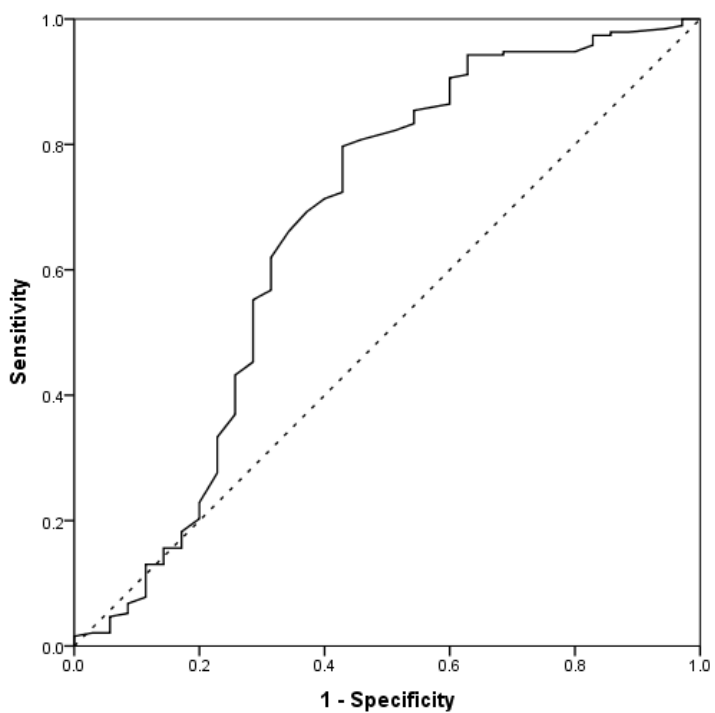

Figure 1: ROC curve analysis of GRACE score for obstructive CAD

$A U C=0.669$ [0.552 to 0.785]; $p=0.001$

The optimal cut-off value based on Youden's index in $\geq 84$

GRACE score of $\geq 84$ was found to be an independent predictor of obstructive CAD with odds ratio of 4.33 [95\% CI: 1.61 - 11.64; $p=0.004$ ] adjusted for gender, age (years), hypertension, diabetes mellitus, family history of $\mathrm{CAD}$, and smoking. Multivariate binary logistic regression analysis for obstructive $\mathrm{CAD}$ is presented in Table 3.

Table 2: Multivariate binary logistic regression analysis for obstructive CAD

\begin{tabular}{|l|c|c|c|}
\hline Parameter & Odds Ratio (OR) & 95\% CI & P-value \\
\hline Male & 1.12 & $0.46-2.69$ & 0.805 \\
\hline Age (years) & 1.01 & $0.96-1.06$ & 0.686 \\
\hline Hypertension & 4.3 & $1.44-12.88$ & $0.009^{*}$ \\
\hline Diabetes Mellitus & 3.36 & $1.25-9.03$ & $0.016^{*}$ \\
\hline $\begin{array}{l}\text { Positive Family } \\
\text { History of CAD }\end{array}$ & 1.56 & $0.32-7.63$ & 0.583 \\
\hline Smoking & 9.27 & $1.75-49.16$ & $0.009^{*}$ \\
\hline GRACE score $\geq 84$ & 4.33 & $1.61-11.64$ & $0.004^{*}$ \\
\hline Constant & 0.18 & - & 0.212 \\
\hline
\end{tabular}

$C A D=$ coronary artery diseases, $C I=$ confidence interval *significant at $5 \%$

\section{DISCUSSION}

This study was conducted with the aim of assessing the predictive value of GRACE score for predicting obstructive CAD in patients with NSTEMI and it was observed that GRACE score showed moderate accuracy in discriminating obstructive CAD with AUC of 0.669 [95\% CI: 0.552 to $0.785 ; \mathrm{p}=0.001$ ]. The optimal cutoff vale of GRACE score was determined to be $\geq 84$, which was found to be an independent 
predictor of obstructive $\mathrm{CAD}$ with good sensitivity of $79.7 \%$ and moderate specificity of $57.1 \%$. Various studies have observed the similar association between GRACE score and extent of CAD in full spectrum of ACS and also specific to the NSTEMI patients.

A study conducted by Roy SS et al. ${ }^{6}$ included 205 patients with NSTEMI and reported positive correlation between GRACE score and severity of CAD, furthermore, study reported superiority of GRACE score over TIMI score in predicting severity of CAD. Similar finding of superiority of GRACE score over TIMI score has been reported in a comparative study of both the scores for predicting the severity of CAD in another study conducted by Roy SS et al. $^{7}$ Hammami $\mathrm{R}$ et al. $^{5}$ reported their observations from the study of 238 consecutive NSTEMI patients regarding relationship between GRACE score and extent of CAD, a moderate accuracy in predicting obstructed CAD has been reported with AUC of 0.599 and GRACE score has sensitivity of $57 \%$ and specificity of $61.8 \%$ at the cutoff value of 120 . However, weak association was observed between the score and severity of CAD, hence, conclusion was made that the score has better predictive power in discriminating obstructive $\mathrm{CAD}$ but not severity of CAD. Rahman ME et al. ${ }^{8}$ conducted a study of 50 NSTEMI patients and reported positive and significant relationship between GRACE score and coronary artery stenosis. Further at the cutoff value of 135, GRACE score was reported to have sensitive of $82.4 \%$ and specificity of $75.8 \%$ in predicting severe CAD. Similarly, Cakar MA et al. ${ }^{9}$ in their study tertiles 245 NSTEMI patients to low-, intermediate-, and high risk groups and reported significant association of GRACE score with severity and extent of coronary artery stenosis.

A study conducted by Mahmood M et al. ${ }^{10}$ in our local population also reported good predictive value of GRACE and TIMI score in predicting the extent of the disease, however, this study also claimed superiority of GRACE score over TIMI score. Various other studies in the spectrum of acute coronary syndrome had similar observations. ${ }^{1,11-13}$ However, one of the largest study in this context so far by dos Santos Viana $\mathrm{M}$ et al. ${ }^{14}$ have argued the association of GRACE score with extent of anatomical coronary disease before angiogram, study included a total of 733 patients out of which $81 \%$ had obstructive CAD and reported AUC for GRACE score was 0.65 for predicting sever disease and at the cutoff value of 103 it has $67 \%$ and $50 \%$ sensitivity and specificity, further, GRACE score was reported to have weak correlation of 0.36 with SYNTAX score. Therefore, role of GRACE score was recommended to limit to prognostic assessments.
Single center experience and relatively smaller sample size are the key limitations of this study, furthermore, study objective was specific to assessment of utility of GRACE score for predicting presence of obstructive CAD only, therefore, no conclusion could be drown regarding the relationship of GRACE score and severity of CAD.

\section{CONCLUSION}

GRACE score has a moderate predictive value in predicting obstructive CAD in patients with NSTEMI. The optimal cut-off value of 84 is an independent predictor with good sensitivity but moderate specificity in predicting obstructive CAD. Hence, this score can be used as a screening tool but its clinical utility for predicting extent of CAD is limited.

\section{AUTHORS' CONTRIBUTION}

HI, TS, and MK: Concept and design, data acquisition, interpretation, drafting, final approval, and agree to be accountable for all aspects of the work. BA, MK, GSS, $\mathrm{AH}$, and SM: Data acquisition, interpretation, drafting, final approval and agree to be accountable for all aspects of the work.

Conflict of interest: Authors declared no conflict of interest.

\section{REFERENCES}

1. Saha T, Khalequzzaman M, Akanda MA, Saha S, Tushar AZ, Ahmed R, et al. Association of GRACE risk score with angiographic severity of coronary artery disease in patients with ST elevation myocardial infarction. Cardiovasc J. 2015;8(1):30-4.

2. Amsterdam EA, Wenger NK, Brindis RG, Casey DE, Ganiats TG, Holmes DR, et al. 2014 AHA/ACC Guideline for the management of patients with non-ST-elevation acute coronary syndromes: a report of the American College of Cardiology/American Heart Association Task Force on Practice Guidelines. J Am Coll Cardiol. 2014;64:e139-228.

3. Roffi M, Patrono C, Collet JP, Mueller C, Valgimigli M, Andreotti F, et al. 2015 ESC Guidelines for the management of acute coronary syndromes in patients pre-senting without persistent STsegment elevation: Task Force for the Management of Acute Coronary Syndromes in Patients Presenting without Persistent STSegment Elevation of the Euro-pean Society of Cardiology (ESC). Eur Heart J. 2016;37:267-315.

4. Antman EM, Cohen M, Bernink PJ, McCabe CH, Horacek T, Papuchis G, et al. The TIMI risk score forunstable angina/non-ST elevation MI: a method for prognostica-tion and therapeutic decision making. JAMA. 2000;284:835-42.

5. Hammami R, Jdidi J, Mroua F, Kallel R, Hentati M, Abid L, et al. Accuracy of the TIMI and GRACE scores in predicting coronary disease in patients with non-ST-elevation acute coronary syndrome. Rev Port Cardiol. 2018;37(1):41-9.

6. Roy SS, Azam SA, Khalequzzaman M, Ullah M, Rahman MA. GRACE and TIMI risk scores in predicting the angiographic severity of non-ST elevation acute coronary syndrome. Indian Heart J. 2018;70:S250-3.

7. Roy SS, Azam SA, Khalequzzaman M, Ullah M, Kundu SK, Hossain MA, et al. Comparison of the GRACE and TIMI Risk Scores in Predicting the Angiographic Severity of Coronary Artery Disease in Patients with non STelevation Myocardial Infarction. Cardiovasc J. 2017;10(1):45-51. 
8. Rahman ME, Safiuddin M, Habib SA, Ahmed CM, Banerjee SK. Association of GRACE Risk Score with Angiographic Severity of Coronary Artery Disease in Patients with Non ST-elevation Acute Coronary Syndrome. Uni Heart J. 2021;17(1):38-41.

9. Cakar MA, Sahinkus S, Aydin E, Vatan MB, Keser N, Akdemir $\mathrm{R}$, et al. Relation between the GRACE score and severity of atherosclerosis in acute coronary syndrome. J Cardiol. 2014;63(1):24-8.

10. Mahmood M, Achakzai AS, Akhtar P, Zaman KS. Comparison of the TIMI and the GRACE risk scores with the extent of coronary artery disease in patients with non-ST-elevation acute coronary syndrome. Heart. 2013;80:91-5.

11. Barbosa CE, Viana M, Brito M, Sabino M, Garcia G, Maraux M, et al. Accuracy of the GRACE and TIMI scores in predicting the angiographic severity of acute coronary syndrome. Arq Bras Cardiol. 2012;99(3):818-24

12. Bekler A, Altun B, Gazi E, Temiz A, Barutçu A, Güngör Ö, et al. Comparison of the GRACE risk score and the TIMI risk index in predicting the extent and severity of coronary artery disease in patients with acute coronary syndrome. Anatol J Cardiol. 2015;15(10):801.

13. Khandelwal G, Jain A, Rathore M. Prediction of angiographic extent of coronary artery disease on the basis of clinical risk scores in patients of unstable angina. Journal of clinical and diagnostic research: JCDR. 2015;9(11):OC13.

14. dos Santos Viana M, Silva TE, Bagano GO, Pontes BD, de Melo MH, Filgueiras PH, et al. Lack of Accuracy of the GRACE score to Predict Coronary Anatomy in Acute Coronary Syndromes. medRxiv. 2021. [PREPRINT].

\section{Address for Correspondence:}

Dr. Haroon Ishaq, Senior Registrar at National Institute of Cardiovascular Diseases (NICVD), Karachi, Pakistan. Email: haroon.ishaq81@gmail.com 